\title{
ON A KAKEYA-TYPE PROBLEM
}

Gregory A. Freiman \& Yonutz V. Stanchescu

Dedicated with best wishes

to Professor Jean-Marc Deshouillers

on the occasion of his 60 th birthday

Abstract: Let $A$ be a finite subset of an abelian group $G$. For every element $b_{i}$ of the sumset $2 A=\left\{b_{0}, b_{1}, \ldots, b_{|2 A|-1}\right\}$ we denote by $D_{i}=\left\{a-a^{\prime}: a, a^{\prime} \in A ; a+a^{\prime}=b_{i}\right\}$ and $r_{i}=\mid\left\{\left(a, a^{\prime}\right)\right.$ : $\left.a+a^{\prime}=b_{i} ; a, a^{\prime} \in A\right\} \mid$. After an eventual reordering of $2 A$, we may assume that $r_{0} \geqslant r_{1} \geqslant \ldots \geqslant r_{|2 A|-1}$. For every $1 \leqslant s \leqslant|2 A|$ we define $R_{s}(A)=\left|D_{0} \cup D_{1} \cup \ldots \cup D_{s-1}\right|$ and $R_{s}(k)=\max \left\{R_{s}(A): A \subset G\right.$, $|A|=k\}$. Bourgain and Katz and Tao obtained an estimate of $R_{s}(k)$ assuming $s$ being of order $k$. In this note we find the exact value of $R_{s}(k)$ in cases $s=1, s=2$ and $s=3$. The case $s=3$ appeared to be not simple. The structure of extremal sets led us to sets isomorphic to planar sets having a rather unexpected form of a perfect hexagon. The proof suggests the way of dealing with the general case $s \geqslant 4$.

Keywords: inverse additive number theory; Kakeya problem.

\section{Introduction}

Let $A$ be a finite subset of an abelian group $(G,+)$. Assume

$$
A=\left\{x_{0}, x_{1}, x_{2}, \ldots, x_{k-1}\right\}, k=|A| .
$$

For every element $b_{i}$ of the sumset

$$
2 A=A+A=\left\{x+x^{\prime}: x \in A, x^{\prime} \in A\right\}=\left\{b_{0}, b_{1}, b_{2}, \ldots, b_{|2 A|-1}\right\}
$$

we denote

$$
\begin{aligned}
r_{i} & =r_{i}(A)=\left|\left\{\left(a, a^{\prime}\right): a+a^{\prime}=b_{i}, a \in A, a^{\prime} \in A\right\}\right|, \\
D_{i} & =D_{i}(A)=\left\{a-a^{\prime}: a \in A, a^{\prime} \in A, a+a^{\prime}=b_{i}\right\}, \\
d_{i} & =d_{i}(A)=\left|D_{i}(A)\right| .
\end{aligned}
$$

After an eventual reordering of the set $2 A$, we may assume that

$$
r_{0} \geqslant r_{1} \geqslant r_{2} \geqslant r_{3} \geqslant \ldots \geqslant r_{|2 A|-1} \text {. }
$$

2000 Mathematics Subject Classification: primary 11P70; secondary 11B75. 
For every $1 \leqslant s \leqslant|2 A|$ we denote

$$
\begin{aligned}
& R_{s}(A)=\left|D_{0} \cup D_{1} \cup D_{2} \cup \ldots \cup D_{s-1}\right|, \\
& R_{s}(k)=\max \left\{R_{s}(A): A \subseteq G,|A|=k\right\} .
\end{aligned}
$$

We shall consider the problem of bounding the quantity $R_{s}(A)$, for sets $A$ included in $\mathbb{Z}^{n}$. Without any further assumption on $s$ one can only obtain the trivial bound $R_{s}(A) \leqslant|A-A| \leqslant k^{2}-k+1$. However, Bourgain [1] proved that under the additional assumption $s \leqslant k$ we have

$$
\left|R_{s}(A)\right| \leqslant k^{2-\frac{1}{13}}
$$

This nontrivial upper bound was improved by Katz and Tao [2] who showed that

$$
\left|R_{s}(A)\right| \leqslant k^{2-\frac{1}{6}}
$$

These estimates were established with an application in mind: by Bourgain and Katz-Tao methods estimates (5) and (6) imply lower bounds for Minkowski and Hausdorff dimension of Kakeya sets.

We would like to find a sharp upper estimate for $R_{s}(k)$ in terms of $k$ and $s$ and to determine its maximal value. Moreover, in cases when the maximal value is obtained, we would like to describe the structure of extremal sets $A^{*}$ for which we have $R_{s}\left(A^{*}\right)=R_{s}(k)$.

In this note we study these questions for the values $s=1, s=2$ and $s=3$. The cases $s=1$ and $s=2$ are straightforward and are proved in Section 2. The case $s=3$ appeared to be not so simple; the structure of extremal sets led us to sets of lattice points in the plane having a rather unexpected form of a perfect hexagon. This is shown in Section 3. Moreover, the proof of this result suggests the way of dealing with the general case. We should mention that the main result we obtained is natural and may be further developed; in Section 4 we discuss briefly some final remarks and open questions. Nevertheless, for the moment, the main result is not connected to the study of dimension of Kakeya sets.

\section{The cases $s=1$ and $s=2$}

Let us begin with some simple remarks, for a finite set $A \subseteq \mathbb{Z}$. We easily see that $d_{i}=r_{i}$ for every $0 \leqslant i \leqslant|2 A|-1$. Indeed, using (2) and (3) we get that for two pairs $\left(a_{1}, a_{1}^{\prime}\right)$ and $\left(a_{2}, a_{2}^{\prime}\right)$ of $A \times A$ such that $a_{1}+a_{1}^{\prime}=a_{2}+a_{2}^{\prime}=b_{i}$ we have $a_{1}-a_{1}^{\prime}=a_{2}-a_{2}^{\prime}$ if and only if the equality $\left(a_{1}, a_{1}^{\prime}\right)=\left(a_{2}, a_{2}^{\prime}\right)$ holds.

Using definition (3), we see that $d_{i}$ is equal to the number of pairs $\left(a, a^{\prime}\right)$ such that $a, a^{\prime} \in A$, and $a$ and $a^{\prime}$ are symmetric with respect to $c_{i}=\frac{b_{i}}{2}$. Moreover, we note that if $a \neq a^{\prime}$ then the pairs $\left(a, a^{\prime}\right)$ and $\left(a^{\prime}, a\right)$ give two distinct differences

$$
a-a^{\prime}=a-\left(b_{i}-a\right)=2 a-b_{i} \quad \text { and } \quad a^{\prime}-a=-\left(2 a-b_{i}\right)
$$

and if $a=a^{\prime}$ we have one pair $(a, a)$ and one difference $d=a-a=0$. 
2.1. Let us look at the case $s=1$. In this situation we have representations

$$
b_{0}=a+a^{\prime} \text {, such that } a \in A \text { and } a^{\prime} \in A
$$

and we count the number $d_{0}=\left|D_{0}(A)\right|$ of all distinct differences $a-a^{\prime}$. Using the above remark, we see that $d_{0}$ cannot exceed the number of elements of $A$, that is $d_{0} \leqslant k=|A|$ and we have equality $d_{0}=k$ if and only if $A$ is a union of pair of points $a, a^{\prime}$ symmetric with respect to $c_{0}=\frac{b_{0}}{2}$. We proved the following

Proposition 1. (a) For every set $A \subseteq \mathbb{Z},|A|=k$ we have

$$
R_{1}(A)=\left|D_{0}(A)\right| \leqslant k
$$

(b) $A$ set of integers $A \subseteq \mathbb{Z}$ satisfies $R_{1}(A)=k$ if and only if $A$ is symmetric with respect to the point $c_{0}=\frac{b_{0}}{2}=\frac{1}{2}(\min A+\max A)$.

2.2. The case $s=2$. We prove the following statement:

Proposition 2. (a) For every set $A \subseteq \mathbb{Z},|A|=k$ we have

$$
R_{2}(A)=\left|D_{0}(A) \cup D_{1}(A)\right| \leqslant 2 k-1
$$

(b) $A$ set of integers $A \subseteq \mathbb{Z}$ satisfies $R_{2}(A)=2 k-1$ if and only if $A$ is an arithmetic progression.

Proof. (a) Denote $2 A=\left\{b_{0}, b_{1}, \ldots, b_{|2 A|-1}\right\}$ and assume that $r_{0} \geqslant r_{1} \geqslant \ldots$. $\geqslant$ $r_{|2 A|-1}$. In view of $r_{1} \leqslant r_{0} \leqslant k$ we should examine only two cases.

(i) If $r_{0} \leqslant k-1$, then $r_{0}+r_{1} \leqslant 2 r_{0} \leqslant 2 k-2$ and from $d_{0}=r_{0}, d_{1}=r_{1}$ we will get

$$
R_{2}(A)=\left|D_{0}(A) \cup D_{1}(A)\right| \leqslant d_{0}+d_{1}=r_{0}+r_{1} \leqslant 2 k-2 .
$$

(ii) If $r_{0}=k$, then $A$ is symmetric with respect to the point $c_{0}=\frac{b_{0}}{2}=$ $\frac{1}{2}(\min A+\max A)$. But $A$ is a finite set and so $c_{1}=\frac{b_{1}}{2}$ cannot be another center of symmetry. This is equivalent to $r_{1} \leqslant k-1$. Consequently if $r_{0}=k$, then $r_{1} \leqslant k-1$ and (a) follows:

$$
R_{2}(A)=\left|D_{0}(A) \cup D_{1}(A)\right| \leqslant d_{0}+d_{1}=r_{0}+r_{1} \leqslant k+(k-1)=2 k-1 .
$$

(b) In order to prove the second assertion of Proposition 2, we first show that for an arithmetic progression $A^{*}=\{1,2,3, \ldots, k\}$ we have

$$
\begin{aligned}
& r_{0}=k, \quad r_{1}=k-1, \\
& D_{0}\left(A^{*}\right) \cap D_{1}\left(A^{*}\right)=\emptyset, \quad R_{2}\left(A^{*}\right)=\left|D_{0}\left(A^{*}\right) \cup D_{1}\left(A^{*}\right)\right|=2 k-1 .
\end{aligned}
$$

It is obvious that $b_{0}=k+1, r_{0}=k$ and $b_{1}=k, r_{1}=k-1$. The sets $D_{0}\left(A^{*}\right)$ and $D_{1}\left(A^{*}\right)$ are disjoint. Indeed, (7) implies that every difference $d_{0} \in D_{0}\left(A^{*}\right)$ is of the form $d_{0}=2 a-b_{0}=2 a-k-1, a \in A^{*}$, every difference $d_{1} \in D_{1}\left(A^{*}\right)$ is of the form $d_{1}=2 a^{*}-b_{1}=2 a^{*}-k, a^{*} \in A^{*}$ and it follows that $2 a-k-1 \not \equiv 2 a^{*}-k$ 
$(\bmod 2)$, that is $D_{0}\left(A^{*}\right) \cap D_{1}\left(A^{*}\right)=\emptyset$. We get that $R_{2}\left(A^{*}\right)=\left|D_{0}\left(A^{*}\right) \cup D_{1}\left(A^{*}\right)\right|=$ $d_{0}+d_{1}=r_{0}+r_{1}=2 k-1$ and (8) follows. verifies

It remains to show that if a set of integers $A=\left\{x_{0}<x_{1}<\ldots<x_{k-1}\right\}$

$$
R_{2}(A)=\left|D_{0}(A) \cup D_{1}(A)\right|=2 k-1,
$$

then $A$ is an arithmetic progression of difference $d=\left|b_{1}-b_{0}\right|$. Assumption (9) implies that $d_{0}=r_{0}=k$ and $d_{1}=r_{1}=k-1$. By Proposition $1, A$ is a symmetric set with respect to $c_{0}=\frac{1}{2} b_{0}$ and thus

$$
b_{0}=x_{0}+x_{k-1}=x_{1}+x_{k-2}=x_{2}+x_{k-3}=\ldots=x_{k-1}+x_{0} .
$$

It is sufficient to examine only the case $b_{0}<b_{1}$. Remark that all $k-1$ solutions of the equation $b_{1}=a+a^{\prime},\left(a, a^{\prime}\right) \in A \times A$ are exactly the pairs $\left(a, b_{1}-a\right)$ with $a \in A \backslash\left\{x_{0}\right\}$. Note that $x_{1}<x_{2}<\ldots<x_{k-1}$ implies $b_{1}-x_{1}>b_{1}-x_{2}>\ldots>$ $b_{1}-x_{k-1}$ and thus

$$
\left\{x_{1}, x_{2}, \ldots, x_{k-1}\right\}=\left\{b_{1}-x_{1}, b_{1}-x_{2}, \ldots, b_{1}-x_{k-1}\right\} .
$$

This implies $x_{1}=b_{1}-x_{k-1}, x_{2}=b_{1}-x_{k-2}, \ldots, x_{k-2}=b_{1}-x_{2}, x_{k-1}=b_{1}-x_{1}$. Using (10) it follows that $x_{1}=b_{0}-x_{k-2}, x_{2}=b_{0}-x_{k-3}, \ldots, x_{k-1}=b_{0}-x_{0}$ and thus

$$
\begin{aligned}
& x_{1}=b_{1}-x_{k-1}=b_{0}-x_{k-2}, \\
& x_{2}=b_{1}-x_{k-2}=b_{0}-x_{k-3}, \ldots, x_{k-1}=b_{1}-x_{1}=b_{0}-x_{0},
\end{aligned}
$$

which is equivalent to $d=b_{1}-b_{0}=x_{1}-x_{0}=x_{2}-x_{1}=x_{3}-x_{2}=\ldots=x_{k-1}-x_{k-2}$. Proposition 2 is proved.

\section{Two-dimensional sets}

In this section we shall study the case $s=3$, i.e. the maximal value of

$$
R_{3}(A)=\left|D_{0}(A) \cup D_{1}(A) \cup D_{2}(A)\right|,
$$

for a finite set $A \subseteq \mathbb{Z}^{2}$ such that $b_{0}, b_{1}, b_{2}$ are non-collinear points. We shall obtain a sharp upper estimate for $R_{3}(A)$ depending only on $k=|A|$; moreover we shall describe the structure of planar extremal sets.

In order to formulate our main result we need some definitions. If $u=$ $\left(u_{1}, u_{2}\right) \in \mathbb{R}^{2}$, we denote by $u_{1}$ and $u_{2}$ its coordinates with respect to the canonical basis $e_{1}=(1,0), e_{2}=(0,1)$ and $e_{0}=(0,0)$ represents the origin point. Let $B$ and $C$ be finite subsets of $\mathbb{Z}^{2}$. We say that $B$ is isomorphic to $C$ if there is an affine isomorphism $L: \mathbb{R}^{2} \rightarrow \mathbb{R}^{2}$ such that $L(B)=C$. 
Let $\alpha \in \mathbb{N}$. We denote by $H(\alpha)$ the set of all points $P=(x, y) \in \mathbb{Z}^{2}$ such that:
(a) $-2 \alpha<x<2 \alpha$,
(b) $-2 \alpha<y<2 \alpha$,
(c) $-2 \alpha<x+y-1<2 \alpha$,
(d) $x$ and $y$ are odd integers.

Note that the convex hull of $H(\alpha)$ is a hexagon (see Figure 1) and its points lie on $2 \alpha$ lines parallel to the line $y=0$, on $2 \alpha$ lines parallel to the line $x=0$ and on $2 \alpha$ lines parallel to the line $x+y=-1$.

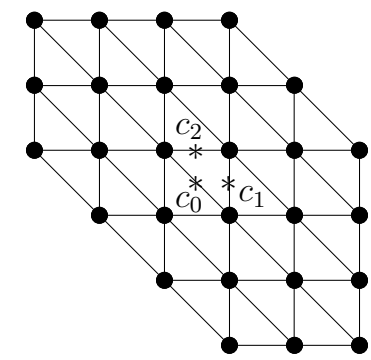

Figure 1. The set $H(\alpha), \alpha=3 ; \quad c_{i}=e_{i}, i=0,1,2$.

We will prove the following

Theorem 1. Let $A$ be a finite subset of $\mathbb{Z}^{2},|A|=k$. Then

(a) $R_{3}(A) \leqslant 3 k-\sqrt{3 k}$ and

(b) $R_{3}(A)=3 k-\sqrt{3 k}$ if and only if $k=3 \alpha^{2}$ and $A$ is isomorphic to $H(\alpha)$.

We shall prove assertion (a) of Theorem 1 in Sections 3.1-3.3 below, while the proof of assertion (b) will be postponed to Section 3.4.

3.1. We study first a set $S \subseteq \mathbb{Q}^{2}$ that lies on two parallel lines. Assume that

$$
\begin{aligned}
S & =S_{+} \cup S_{-}, \\
S_{+} & =\left\{P_{1}, \ldots, P_{s_{+}}\right\}, P_{i}=\left(a_{i}, 1\right), a_{1}<a_{2}<\ldots<a_{s_{+}}, \\
S_{-} & =\left\{Q_{1}, \ldots, Q_{s_{-}}\right\}, Q_{i}=\left(b_{i},-1\right), b_{1}<b_{2}<\ldots<b_{s_{-}}
\end{aligned}
$$

and thus

$$
\begin{aligned}
S_{+} & =S \cap(y=1), \quad s_{+}=\left|S_{+}\right|, \quad S_{-}=S \cap(y=-1), \quad s_{-}=\left|S_{-}\right|, \\
s & =|S|=s_{+}+s_{-} .
\end{aligned}
$$

Denote by $A=\left\{a_{1}<\ldots<a_{s_{+}}\right\}, B=\left\{b_{1}<\ldots<b_{s_{-}}\right\}$the sets of abscissae of $S_{+}$ and $S_{-}$, respectively. We study the sets of differences

$$
\begin{aligned}
& D_{0}(S)=\left\{P-P^{\prime}: P \in S, P^{\prime} \in S, P+P^{\prime}=e_{0}\right\}, e_{0}=(0,0), \\
& D_{1}(S)=\left\{P-P^{\prime}: P \in S, P^{\prime} \in S, P+P^{\prime}=e_{1}\right\}, e_{1}=(1,0) .
\end{aligned}
$$


If $P_{i}=e_{i}-P$ is the symmetric of $P$ with respect to $\frac{1}{2} e_{i}$, then

$$
D_{i}(S)=\left\{2 P-e_{i}: P \in S, P_{i} \in S\right\}, i=0,1 .
$$

\section{Lemma 1.}

(a) $\left|D_{i}(S)\right| \leqslant 2 \min \left\{s_{+}, s_{-}\right\}$, for $i=1,2$.

(b) $\left|D_{0}(S) \cup D_{1}(S)\right| \leqslant\left|D_{0}(S)\right|+\left|D_{1}(S)\right| \leqslant 2|S|-2$.

(c) If $\left|D_{0}(S) \cup D_{1}(S)\right|=2|S|-2$, then the set $S$ consists of two parallel arithmetic progressions of common difference $e_{1}-e_{0}$.

Proof. (a) Let us assume that $s_{+} \leqslant s_{-}$. Each point $P \in S_{+}$defines no more than two differences $d=P-P_{i}$ or $-d=P_{i}-P$ and so

$$
D_{i}(S) \subseteq\left\{ \pm\left(P-P^{\prime}\right): P \in S_{+}, P+P^{\prime}=e_{i}\right\} .
$$

This implies that $\left|D_{i}(S)\right| \leqslant 2 s_{+}$. Similarly, if $s_{-} \leqslant s_{+}$, then $\left|D_{i}(S)\right| \leqslant 2 s_{-}$. Therefore, in both cases we obtain $\left|D_{i}(S)\right| \leqslant 2 \min \left\{s_{+}, s_{-}\right\}$.

(b) Using inequality

$$
\left|D_{i}(S)\right| \leqslant 2 \min \left\{s_{+}, s_{-}\right\}=|S|-\left|s_{+}-s_{-}\right| \leqslant|S|,
$$

we get that if $\left|s_{+}-s_{-}\right| \geqslant 1$ then clearly (b) is true. It remains to consider only the case $s_{+}=s_{-}$. Without loss of generality we may assume that $\left|D_{1}(S)\right| \leqslant\left|D_{0}(S)\right|$. We shall distinguish two cases.

Case (i). If there is a point $R$ in $S_{+}$such that $R_{0}=e_{0}-R$ is not in $S_{-}$, then the differences $\pm d= \pm\left(R-R_{0}\right)$ don't belong to $D_{0}(S)$. Therefore in this case we get $\left|D_{1}(S)\right| \leqslant\left|D_{0}(S)\right| \leqslant 2\left(s_{+}-1\right)=|S|-2$ and so $\left|D_{0}(S)\right|+\left|D_{1}(S)\right| \leqslant 2|S|-4$.

Case (ii). Assume that for every point $P \in S_{+}$, its symmetric with respect to $\frac{1}{2} e_{0}$ belongs to $S_{-}$, that is $P_{0}=e_{0}-P \in S_{-}$. Using $s_{+}=s_{-}$we get that $S_{+}$ and $S_{-}$are symmetric with respect to $\frac{1}{2} e_{0}$. In this case we have $\left|D_{0}(S)\right|=2 s_{+}=$ $2 s_{-}=|S|$ and in order to prove (b) it remains to show that $\left|D_{1}(S)\right| \leqslant|S|-2$. The set $S_{+}$lies in the segment determined by the points $P_{1}$ and $P_{s_{+}}$. The set $S_{-}$lies in the segment determined by the points $Q_{1}=e_{0}-P_{s_{+}}$and $Q_{s_{-}}=e_{0}-P_{1}$. In consequence, $e_{1}-P_{1}$, the symmetric of $P_{1}$ with respect to $\frac{1}{2} e_{1}$ does not belong to $S_{-}$. Similarly, $e_{1}-Q_{1}$, the symmetric of $Q_{1}$ with respect to $\frac{1}{2} e_{1}$ does not belong to $S_{+}$. Therefore, $\left|D_{1}(S)\right| \leqslant|S|-2$. where

(c) Let $i=0$ or $i=1$. Note that $D_{i}$ is the disjoint union of $D_{i}=D_{i}^{+} \cup D_{i}^{-}$,

$$
\begin{aligned}
D_{i}^{+} & =\left\{P-P_{i}: P \in S_{+}, P_{i} \in S_{-}\right\}=\left\{\delta=\left(\delta_{1}, \delta_{2}\right) \in D_{i}: \delta_{2}=+2\right\}, \\
D_{i}^{-} & =\left\{P-P_{i}: P \in S_{-}, P_{i} \in S_{+}\right\}=\left\{\delta=\left(\delta_{1}, \delta_{2}\right) \in D_{i}: \delta_{2}=-2\right\}, \\
d_{i}^{+} & =\left|D_{i}^{+}\right|=d_{i}^{-}=\left|D_{i}^{-}\right| .
\end{aligned}
$$

Moreover, each difference belonging to $D_{i}^{+}$can be written as

$$
\delta^{+}=P-P_{i}=(x, 1)-(y,-1)=(x-y, 2), \text { with } x \in A, y \in B, x+y=i .
$$


Therefore, $x \in A \cap(i-B), d_{i}^{+}=|A \cap(i-B)|$ and

$$
d_{i}=\left|D_{i}\right|=d_{i}^{+}+d_{i}^{-}=2 d_{i}^{+}=2|A \cap(i-B)| .
$$

Without loss of generality we may assume that $\left|D_{1}(S)\right| \leqslant\left|D_{0}(S)\right|$. We need to examine only two cases:

Case (i). Assume that $d_{0}=\left|D_{0}(S)\right|=|S|>d_{1}=\left|D_{1}(S)\right|=|S|-2$. If $t=|A \cap(-B)|$ then

$$
|A|+|B|=|S|=d_{0}=2 d_{0}^{+}=2|A \cap(-B)|=2 t \leqslant 2 \min (|A|,|B|)
$$

and thus $|A \cap(-B)|=|A|=|B|=t$. We get that $B=-A$, i.e. $S$ is a symmetric set with respect to $\frac{e_{0}}{2}$. It follows that $2 t-2=|S|-2=d_{1}=2 d_{1}^{+}=2|A \cap(1-B)|=$ $2|A \cap(1+A)|$, and so $|A \cap(1+A)|=t-1=|A|-1$. We conclude that $S$ consists of two arithmetic progressions $S_{+}$and $S_{-}$, with $S_{-}=-S_{+}$.

Case (ii). Assume that $\left|D_{0}(S)\right|=\left|D_{1}(S)\right|=|S|-1$. It $t=|A \cap(-B)|$ then

$|S|-1=d_{0}=2 d_{0}^{+}=2|A \cap(-B)|=2 t$ and $2 t=|S|-1=d_{1}=2 d_{1}^{+}=2|A \cap(1-B)|$.

We get that $|A \cap(-B)|=|A \cap(1-B)|=t,|A| \geqslant t,|B| \geqslant t,|S|=|A|+|B|=2 t+1$. If $|A|=t$, then $|B|=t+1$ and $A, B$ are arithmetic progression such that $B \backslash\left\{b_{s_{-}}\right\}=-A$. If $|A|=t+1$, then $|B|=t$ and $A, B$ are arithmetic progression such that $A \backslash\left\{a_{s_{+}}\right\}=-B$. Lemma 1 is proved.

3.2. Let $A \subseteq \mathbb{Q}^{2}$ be a finite set, $|A|=k$. Using an affine isomorphism that maps the points $b_{0}, b_{1}, b_{2}$ onto the standard simplex $e_{0}=(0,0), e_{1}=(1,0), e_{2}=(0,1)$, we may assume, without loss in generality that

$$
A \subseteq \mathbb{Q}^{2}, b_{0}=e_{0}=(0,0), b_{1}=e_{1}=(1,0), b_{2}=e_{2}=(0,1) .
$$

In this section we shall generalize Lemma 1 for sets lying on $a$ lines parallel to $y=0$ and we will estimate the cardinality of

$$
\operatorname{Diff}(A)=D_{0} \cup D_{1} \cup D_{2},
$$

where

$$
D_{i}=D_{i}(A)=\left\{P-P^{\prime}: P \in A, P^{\prime} \in A, P+P^{\prime}=e_{i}\right\}, \quad i=0,1,2 .
$$

Denote by $P_{i}=e_{i}-P$ the symmetric of $P$ with respect to $\frac{1}{2} e_{i}$. It follows that each set of differences $D_{i}$ satisfies

$$
\begin{aligned}
D_{i} & =\left\{P-P_{i}: P \in A, P_{i} \in A\right\}=\left\{2 P-e_{i}: P \in A, P_{i} \in A\right\}, \\
d_{i}=\left|D_{i}\right| & =\left|\left\{P: P \in A, P_{i} \in A\right\}\right|, \quad i=0,1,2 .
\end{aligned}
$$

For every $h$, we denote by

$$
A_{h}=A \cap(y=h)
$$


the set of points of $A$ that lie on the line $y=h$. The set $D_{i}$ consists of all differences $d=P-P_{i}$ such that both $P$ and $P_{i}$ belong to the set $A$. Note that if $P \in A_{h}$, then $P_{0} \in A_{-h}, P_{1} \in A_{-h}$ and $P_{2} \in A_{-h+1}$. This remark allows us to split $D_{i}$ into a disjoint union of sets:

$$
D_{i}=\bigcup_{h} D_{i}(h)
$$

where

$$
\begin{aligned}
& D_{0}(h)=D_{0}(A, h)=\left\{2 P-e_{0}: P \in A_{h}, P_{0} \in A_{-h}\right\}, \\
& D_{1}(h)=D_{1}(A, h)=\left\{2 P-e_{1}: P \in A_{h}, P_{1} \in A_{-h}\right\}, \\
& D_{2}(h)=D_{2}(A, h)=\left\{2 P-e_{2}: P \in A_{h}, P_{2} \in A_{-h+1}\right\} .
\end{aligned}
$$

Let $a$ be the number of lines $\ell^{\prime}$ parallel to the line $y=0$ such that $A \cap \ell^{\prime} \neq \emptyset$. In a similar way, let $b$ be the number of lines $\ell^{\prime \prime}$ parallel to the line $x=0$ such that $A \cap \ell^{\prime \prime} \neq \emptyset$ and $c$ be the number of lines $\ell^{*}$ parallel to the line $x+y=0$ such that $A \cap \ell^{*} \neq \emptyset$.

Lemma 2. (a) $\left|D_{0}(A) \cup D_{1}(A)\right| \leqslant 2 k-a, \quad\left|D_{0}(A) \cup D_{2}(A)\right| \leqslant 2 k-b \quad$ and $\left|D_{1}(A) \cup D_{2}(A)\right| \leqslant 2 k-c$.

(b) $|\operatorname{Diff}(A)|=\left|D_{0}(A) \cup D_{1}(A) \cup D_{2}(A)\right| \leqslant 3 k-\frac{1}{2}(a+b+c)$.

Proof. (a) Let $H$ be the set of all integers $h$ such that $A_{ \pm h}=A_{h} \cup A_{-h} \neq \emptyset$. We have

$$
\begin{aligned}
D_{0}(A) & =\bigcup_{h \in H} D_{0}(h)=\bigcup_{h \in H, h \geqslant 0} D_{0}\left(A_{ \pm h}\right), \\
D_{1}(A) & =\bigcup_{h \in H} D_{1}(h)=\bigcup_{h \in H, h \geqslant 0} D_{1}\left(A_{ \pm h}\right), \\
D_{0}(A) \cup D_{1}(A) & =\bigcup_{h \in H, h \geqslant 0}\left(D_{0}\left(A_{ \pm h}\right) \cup D_{1}\left(A_{ \pm h}\right)\right) .
\end{aligned}
$$

If $h=0$ belongs to $H$, we have $\left|D_{0}\left(A_{0}\right) \cup D_{1}\left(A_{0}\right)\right| \leqslant 2\left|A_{0}\right|-1$, in view of Proposition 2(a). For $0<h \in H$, the set $A_{ \pm h}$ is included on two parallel lines. If $\left|A_{h}\right|>0$ and $\left|A_{-h}\right|>0$, then by Lemma 1(b), we get $\left|D_{0}\left(A_{ \pm h}\right) \cup D_{1}\left(A_{ \pm h}\right)\right| \leqslant$ $2\left|A_{ \pm h}\right|-2$. If $\left|A_{h}\right|=0$ or $\left|A_{-h}\right|=0$, then $A_{ \pm h}$ lies on a line and obviously $\left|D_{0}\left(A_{ \pm h}\right) \cup D_{1}\left(A_{ \pm h}\right)\right|=0 \leqslant 2\left|A_{ \pm h}\right|-2<2\left|A_{ \pm h}\right|-1$. We conclude that

$$
\begin{aligned}
\left|D_{0}(A) \cup D_{1}(A)\right| & =\sum_{h \in H, h \geqslant 0}\left|D_{0}\left(A_{ \pm h}\right) \cup D_{1}\left(A_{ \pm h}\right)\right| \leqslant \\
& \leqslant\left(2\left|A_{0}\right|-1\right)+\sum_{h \in H, h>0}\left(2\left|A_{ \pm h}\right|-2\right) \leqslant \\
& \leqslant 2|A|-a=2 k-a .
\end{aligned}
$$

Note that this inequality was obtained using a partition of $A$ into sets lying on lines parallel to the segment $\left[e_{0}, e_{1}\right]$. In a similar way, considering lines parallel to the segments $\left[e_{0}, e_{2}\right]$ and $\left[e_{1}, e_{2}\right]$, we obtain respectively that

$$
\left|D_{0}(A) \cup D_{2}(A)\right| \leqslant 2 k-b \text { and }\left|D_{1}(A) \cup D_{2}(A)\right| \leqslant 2 k-c .
$$


(b) Denote by

$$
d_{i j}=\left|D_{i}(A) \cap D_{j}(A)\right|, d_{012}=\left|D_{0}(A) \cap D_{1}(A) \cap D_{2}(A)\right| .
$$

Then $\left|D_{0}(A) \cup D_{1}(A) \cup D_{2}(A)\right|=\left(d_{0}+d_{1}+d_{2}\right)-\left(d_{01}+d_{02}+d_{12}\right)+d_{012}$ and thus

$$
\begin{aligned}
2 \mid & D_{0}(A) \cup D_{1}(A) \cup D_{2}(A) \mid= \\
= & \left(d_{0}+d_{1}-d_{01}\right)+\left(d_{0}+d_{2}-d_{02}\right)+\left(d_{1}+d_{2}-d_{12}\right) \\
& -d_{01}-d_{02}-d_{12}+2 d_{012} \\
= & \left|D_{0}(A) \cup D_{1}(A)\right|+\left|D_{0}(A) \cup D_{2}(A)\right|+\left|D_{1}(A) \cup D_{2}(A)\right| \\
& -d_{01}-d_{02}-d_{12}+2 d_{012} \\
\leqslant & 6 k-(a+b+c)-d_{i j} .
\end{aligned}
$$

We conclude that for every $0 \leqslant i \neq j \leqslant 2$

$$
\begin{aligned}
|\operatorname{Diff}(A)| & =\left|D_{0}(A) \cup D_{1}(A) \cup D_{2}(A)\right| \leqslant 3 k-\frac{a+b+c}{2}-\frac{d_{i j}}{2} \\
& \leqslant 3 k-\frac{a+b+c}{2} .
\end{aligned}
$$

Lemma 2 is proved.

Example 1. Inequality (16) is sharp. The set

$$
A^{*}=\frac{1}{2} H(\alpha)=\left\{\frac{1}{2}(x, y):(x, y) \in H(\alpha)\right\}
$$

satisfies $a=b=c=2 \alpha,\left|D_{i}\left(A^{*}\right)\right|=k-\alpha$, for $i=0,1,2$ and $\left|\operatorname{Diff}\left(A^{*}\right)\right|=$ $\left|D_{0}\left(A^{*}\right)\right|+\left|D_{1}\left(A^{*}\right)\right|+\left|D_{2}\left(A^{*}\right)\right|=3 k-3 \alpha=3 k-\frac{a+b+c}{2}$; therefore inequality (16) cannot be sharpened by reducing the upper bound for $R_{3}\left(A^{*}\right)$.

3.3. In this section we obtain the following optimal upper bound

$$
|\operatorname{Diff}(A)|=\left|D_{0}(A) \cup D_{1}(A) \cup D_{2}(A)\right| \leqslant 3 k-\sqrt{3 k},
$$

valid for every finite set $A \subseteq \mathbb{Q}^{2},|A|=k$.

We shall first estimate the cardinality of a finite set $K \subseteq \mathbb{Q}^{2}$ assuming that $K$ lies on $a$ lines parallel to the line $y=0$, on $b$ lines parallel to the line $x=0$ and on $c$ lines parallel to the line $x+y=0$.

Lemma 3. Let $K$ be a finite subset of $\mathbb{Q}^{2}$. If $\max \{a, b, c\}<\frac{a+b+c}{2}$, then

$$
k=|K| \leqslant \frac{1}{3} \frac{(a+b+c)^{2}}{4}+\frac{\delta}{4}
$$

where $\delta=0$ if $a+b+c$ is even and $\delta=1$, if $a+b+c$ is odd.

Proof. We clearly have $k \leqslant \frac{a b c}{\max \{a, b, c\}}$. Moreover, let us note that if $\max \{a, b, c\} \geqslant$ $\frac{a+b+c}{2}$, then $k \leqslant \frac{\max ^{2}\{a, b, c\}}{4}$. Indeed, assuming that $a=\max \{a, b, c\}$ we get $a \geqslant b+c$ and thus

$$
k \leqslant b c \leqslant \frac{(b+c)^{2}}{4} \leqslant \frac{a^{2}}{4}=\frac{\max ^{2}\{a, b, c\}}{4} .
$$


In order to prove Lemma 3 , we shall apply induction on $\min \{a, b, c\}$.

If $\min \{a, b, c\}=a=1$, then $K$ lies on a line, $|K|=k, a=1, b=c=k$, $a+b+c=1+2 k$ is odd and thus $\delta=1$. In this case Lemma 3 is true because

$$
\frac{1}{3} \frac{(a+b+c)^{2}}{4}+\frac{\delta}{4}=\frac{1}{3} \frac{(2 k+1)^{2}}{4}+\frac{1}{4}=\frac{1}{3}\left(k^{2}+k+1\right)=\frac{1}{3}(k-1)^{2}+k \geqslant k .
$$

Let us assume that $\min \{a, b, c\} \geqslant 2$ and the assertion holds for smaller values of $\min \{a, b, c\}$. There is no loss in generality if we assume that $a \geqslant b \geqslant c$. Note that $\max \{a, b, c\}<\frac{a+b+c}{2}$ implies

$$
b+c>a \geqslant b \geqslant c .
$$

Let us choose (see Figure 2)

$$
\ell_{a}: x=u^{\prime}, \ell_{a}^{\prime}: x+y=w^{\prime} ; \quad \ell_{c}: y=v^{\prime}, \ell_{c}^{\prime}: x=u ; \quad \ell_{b}: x+y=w, \ell_{b}^{\prime}: y=v
$$

supporting lines of the convex hull of $K$ such that $K$ is included in the half planes

$$
x \geqslant u^{\prime}, x+y \geqslant w^{\prime}, y \geqslant v^{\prime}, x \leqslant u, x+y \leqslant w, y \leqslant v .
$$

Denote by

$$
K_{a}=K \backslash\left(\ell_{a} \cup \ell_{a}^{\prime}\right), K_{b}=K \backslash\left(\ell_{b} \cup \ell_{b}^{\prime}\right), K_{c}=K \backslash\left(\ell_{c} \cup \ell_{c}^{\prime}\right)
$$

and

$$
K^{\prime}=K \backslash\left(\ell_{a} \cup \ell_{a}^{\prime} \cup \ell_{b} \cup \ell_{b}^{\prime} \cup \ell_{c} \cup \ell_{c}^{\prime}\right) .
$$

We have $|K| \leqslant\left|K_{a}\right|+a,|K| \leqslant\left|K_{b}\right|+b,|K| \leqslant\left|K_{c}\right|+c$ and thus

$$
|K| \leqslant\left|K^{\prime}\right|+(a+b+c-3) .
$$

Clearly, the set $K^{\prime}$ lies on $a^{\prime} \leqslant a-2$ lines parallel to $y=0$, on $b^{\prime} \leqslant b-2$ lines parallel to $x=0$ and on $c^{\prime} \leqslant c-2$ lines parallel to $x+y=0$. We denote $s^{\prime}=a^{\prime}+b^{\prime}+c^{\prime}$ and $s=a+b+c$. Put $\delta^{\prime}=0$ if $a^{\prime}+b^{\prime}+c^{\prime}$ is even and $\delta^{\prime}=1$, if $a^{\prime}+b^{\prime}+c^{\prime}$ is odd.

We shall estimate $|K|$ using the induction hypothesis for the set $K^{\prime} \varsubsetneqq K$.

We distinguish several cases.

Case 1. Assume that $\max \left\{a^{\prime}, b^{\prime}, c^{\prime}\right\}<\frac{a^{\prime}+b^{\prime}+c^{\prime}}{2}$. By the induction hypothesis we get $\left|K^{\prime}\right| \leqslant \frac{1}{3} \frac{\left(a^{\prime}+b^{\prime}+c^{\prime}\right)^{2}}{4}+\frac{\delta^{\prime}}{4}$. Using

$$
s^{\prime} \leqslant s-6
$$

we get

$$
\begin{aligned}
|K| & \leqslant\left|K^{\prime}\right|+(a+b+c-3) \leqslant \frac{1}{3} \frac{s^{2}}{4}+\frac{\delta^{\prime}}{4}+(s-3) \\
& \leqslant \frac{1}{3} \frac{(s-6)^{2}}{4}+\frac{\delta^{\prime}}{4}+(s-3)=\frac{1}{3} \frac{s^{2}}{4}+\frac{\delta^{\prime}}{4} .
\end{aligned}
$$


Case 1.1. If $\delta^{\prime}=0$, then $|K| \leqslant \frac{1}{3} \frac{s^{2}}{4} \leqslant \frac{1}{3} \frac{(a+b+c)^{2}}{4}+\frac{\delta}{4}$.

Case 1.2. If $s^{\prime}=s-6$, then $\delta=\delta^{\prime}$ and we get $|K| \leqslant \frac{1}{3} \frac{(a+b+c)^{2}}{4}+\frac{\delta}{4}$.

Case 1.3. If $\delta^{\prime}=1$ and $s^{\prime} \leqslant s-7$ then $s \geqslant s^{\prime}+7 \geqslant 10$ and thus

$$
\begin{aligned}
|K| & \leqslant \frac{1}{3} \frac{s^{\prime 2}}{4}+\frac{\delta^{\prime}}{4}+(s-3) \leqslant \frac{1}{3} \frac{(s-7)^{2}}{4}+\frac{1}{4}+(s-3)=\frac{1}{3} \frac{s^{2}}{4}-\frac{s}{6}+\frac{4}{3} \\
& <\frac{1}{3} \frac{s^{2}}{4}+\frac{\delta}{4}=\frac{1}{3} \frac{(a+b+c)^{2}}{4}+\frac{\delta}{4} .
\end{aligned}
$$

Case 2. Assume that $\max \left\{a^{\prime}, b^{\prime}, c^{\prime}\right\} \geqslant \frac{a^{\prime}+b^{\prime}+c^{\prime}}{2}$. Using inequality (17) and (19) we get $\left|K^{\prime}\right| \leqslant\left(\frac{\max \left\{a^{\prime}, b^{\prime}, c^{\prime}\right\}}{2}\right)^{2} \leqslant\left(\frac{\max \{a, b, c\}-2}{2}\right)^{2}=\frac{(a-2)^{2}}{4}$ and $|K| \leqslant \frac{(a-2)^{2}}{4}+$ $(a+b+c-3)$. In order to prove inequality $|K| \leqslant \frac{1}{3} \frac{(a+b+c)^{2}}{4}+\frac{\delta}{4}$ it is enough to verify that under the hypothesis (18) the number $\Delta=\frac{(a+b+c)^{2}}{12}+\frac{\delta}{4}-(a+b+c)+3-\frac{(a-2)^{2}}{4}$ is nonnegative, i.e.

$$
\Delta=\frac{(a+b+c-6)^{2}}{12}+\frac{\delta}{4}-\frac{(a-2)^{2}}{4} \geqslant 0 .
$$

Case 2.1. If $a \geqslant 6$ then using $b+c \geqslant a+1$ we get

$$
\Delta \geqslant \frac{(2 a-5)^{2}}{12}+\frac{\delta}{4}-\frac{(a-2)^{2}}{4} \geqslant \frac{(a-4)^{2}-3}{12} \geqslant \frac{1}{12}>0 .
$$

Case 2.2. If $a=5$, then $b+c \geqslant a+1=6$. We distinguish two subcases.

If $b+c \geqslant 7$, then $\Delta=\frac{(b+c-1)^{2}}{12}+\frac{\delta}{4}-\frac{9}{4} \geqslant 3+\frac{\delta}{4}-\frac{9}{4} \geqslant \frac{3}{4}>0$.

If $b+c=6$, then $\delta=1$ and thus $\Delta=\frac{(b+c-1)^{2}}{12}+\frac{\delta}{4}-\frac{9}{4}=\frac{25}{12}+\frac{1}{4}-\frac{9}{4}=\frac{1}{12}>0$.

Case 2.3. If $a=4$, then $b+c \geqslant a+1=5$. We distinguish two subcases.

If $b+c \geqslant 6$, then $\Delta=\frac{(b+c-2)^{2}}{12}+\frac{\delta}{4}-1 \geqslant \frac{16}{12}-1>0$.

If $b+c=5$, then $\delta=1$ and thus $\Delta=\frac{(b+c-2)^{2}}{12}+\frac{\delta}{4}-1=\frac{9}{12}+\frac{1}{4}-1=0$.

Case 2.4. If $a=3$, then $b+c \geqslant a+1=4$. We distinguish two subcases.

If $b+c \geqslant 5$, then $\Delta=\frac{(b+c-3)^{2}}{12}+\frac{\delta}{4}-\frac{1}{4} \geqslant \frac{4}{12}-\frac{1}{4}>0$.

If $b+c=4$, then $\delta=1$ and thus $\Delta=\frac{(b+c-3)^{2}}{12}+\frac{\delta}{4}-\frac{1}{4}=\frac{1}{12}>0$.

Case 2.5. If $a=2$, then $\min \{a, b, c\} \geqslant 2$ implies $b=c=2$ and thus $\Delta=\frac{\delta}{4}=0$.

An easy consequence of Lemma 3 is

Lemma 4. Let $K \subseteq \mathbb{Q}^{2}$ be a finite set such that $\max \{a, b, c\}=m$. We have:

(a) If $m \geqslant \frac{a+b+c}{2}$, then $|K| \leqslant \frac{m^{2}}{4}$.

(b) If $m<\frac{a+b+c}{2}$, then $|K| \leqslant \frac{1}{3}\left(\frac{a+b+c}{2}\right)^{2}+\frac{\delta}{4} \leqslant \frac{3 m^{2}}{4}+\frac{\delta}{4}$, where $\delta=0$ if $a+b+c$ is even and $\delta=1$, if $a+b+c$ is odd.

Proof. (a) Assertion (a) is equivalent to inequality (17). 

obtain

(b) Assume that $c \leqslant b \leqslant a=\max \{a, b, c\}<\frac{a+b+c}{2}$. In view of Lemma 3 we

$$
k=|K| \leqslant \frac{1}{3}\left(\frac{a+b+c}{2}\right)^{2}+\frac{\delta}{4} \leqslant \frac{1}{3} \frac{(3 m)^{2}}{4}+\frac{\delta}{4}=\frac{3 m^{2}}{4}+\frac{\delta}{4} .
$$

Lemma 4 is proved.

Example 2. Lemmas 3 and 4 cannot be sharpened by reducing the upper bound for $|K|$. Indeed, the set $K=H(\alpha)$ satisfies $a=b=c=2 \alpha$ and

$$
k=|K|=3 \alpha^{2}=\frac{1}{3} \frac{(a+b+c)^{2}}{4}=\frac{3 \max ^{2}(a, b, c)}{4} .
$$

Moreover, we may note at this point that Example 1 implies the following equality

$$
R_{3}\left(A^{*}\right)=\left|\operatorname{Diff}\left(A^{*}\right)\right|=3 k-\sqrt{3 k},
$$

valid for the set $A^{*}=\frac{1}{2} H(\alpha)$.

We shall complete this section by proving inequality (a) of Theorem 1; Lemma 3 implies the following

Corollary 1. Let $A$ be a finite subset of $\mathbb{Q}^{2},|A|=k$. Then

$$
R_{3}(A)=|\operatorname{Diff}(A)|=\left|D_{0}(A) \cup D_{1}(A) \cup D_{2}(A)\right| \leqslant 3 k-\sqrt{3 k} .
$$

Proof. In order to prove (25) we shall examine two cases separately.

(i) If $\max \{a, b, c\}<\frac{a+b+c}{2}$, then Lemma 3 implies $k=|A| \leqslant \frac{1}{3}\left(\frac{a+b+c}{2}\right)^{2}+\frac{\delta}{4}$. This is equivalent to $\frac{1}{2}(a+b+c) \geqslant \sqrt{3 k-\frac{3}{4} \delta}$; using Lemma 2 (b) we get

$$
\begin{aligned}
|\operatorname{Diff}(A)| & =\left|D_{0}(A) \cup D_{1}(A) \cup D_{2}(A)\right| \leqslant 3 k-\frac{a+b+c}{2}-\frac{\delta}{2} \\
& \leqslant 3 k-\sqrt{3\left(k-\frac{\delta}{4}\right)}-\frac{\delta}{2} \leqslant 3 k-\sqrt{3 k} .
\end{aligned}
$$

(ii) Assume $\max \{a, b, c\} \geqslant \frac{a+b+c}{2}$ and, without loss of generality, $a=$ $\max \{a, b, c\}$. We get $b+c \leqslant a$ and thus $k=|A| \leqslant b c=\frac{(b+c)^{2}-(b-c)^{2}}{4} \leqslant$ $\frac{\left(\frac{a+b+c}{2}\right)^{2}-(b-c)^{2}}{4}$. It follows that

$$
|\operatorname{Diff}(A)| \leqslant 3 k-\frac{a+b+c}{2} \leqslant 3 k-\sqrt{4 k+(b-c)^{2}} \leqslant 3 k-\sqrt{4 k}<3 k-\sqrt{3 k} .
$$

Corollary 1 is proved.

3.4. In this section we shall prove assertion (b) of Theorem 1. In view of equality (24), it remains to show that if $A$ is an extremal set, i.e.

$$
R_{3}(A)=|\operatorname{Diff}(A)|=3 k-\sqrt{3 k}, k=|A|,
$$


then $A$ is isomorphic to $H(\alpha)$, for $\alpha=\sqrt{\frac{k}{3}}$. Assume that the set $A$ lies on $a$ lines parallel to $y=0$, on $b$ lines parallel to $x=0$ and on $c$ lines parallel to $x+y=0$.

Assertion 1. (i) There is a natural number $\alpha$ such that $k=3 \alpha^{2}$.

(ii) $R_{3}(A)=|\operatorname{Diff}(A)|=3 k-\frac{a+b+c}{2}=3 k-\sqrt{3 k}$.

(iii) $a+b+c=6 \alpha$ and $k=\frac{(a+b+c)^{2}}{12}$.

Proof. In view of (29) and (28) we may assume that there is $\alpha \in \mathbb{N}$ such that

$$
k=3 \alpha^{2} \quad \text { and } \max \{a, b, c\}<\frac{a+b+c}{2} .
$$

From (26), (27) and (29) we get that:

$$
R_{3}(A)=|\operatorname{Diff}(A)|=3 k-\frac{a+b+c}{2}-\frac{\delta}{2}=3 k-\sqrt{3 k-\frac{3}{4} \delta}-\frac{\delta}{2}=3 k-\sqrt{3 k}
$$

If $\delta=1$ then $3 k-\sqrt{3 k-\frac{3}{4} \delta}-\frac{\delta}{2}<3 k-\sqrt{3 k}$, which contradicts (31). Thus $\delta=0$, which means that $a+b+c=2 \sqrt{3 k}=6 \alpha$. We get

$$
R_{3}(A)=|\operatorname{Diff}(A)|=3 k-\frac{a+b+c}{2}=3 k-\sqrt{3 k}, \quad k=\frac{(a+b+c)^{2}}{12}
$$

and so Assertion 1 is proved.

Assertion 2. (i) $\left|D_{0}(A) \cup D_{1}(A)\right|=\left|D_{0}(A)\right|+\left|D_{1}(A)\right|=2 k-a$.

(ii) $\left|D_{0}(A) \cup D_{2}(A)\right|=\left|D_{0}(A)\right|+\left|D_{2}(A)\right|=2 k-b$.

(iii) $\left|D_{1}(A) \cup D_{2}(A)\right|=\left|D_{1}(A)\right|+\left|D_{2}(A)\right|=2 k-c$.

(iv) Let $0 \leqslant i \neq j \leqslant 2$. For every line $\ell$ parallel to the segment $\left[e_{i}, e_{j}\right]$ the set $A \cap \ell$ is an arithmetic progression of difference $e_{i}-e_{j}$.

Proof. Using inequality (16) and equality (ii) of Assertion 1 we get $d_{i j}=\mid D_{i}(A) \cap$ $D_{j}(A) \mid=0$ and thus $\left|D_{i}(A) \cup D_{j}(A)\right|=\left|D_{i}(A)\right|+\left|D_{j}(A)\right|$, for every $0 \leqslant i \neq j \leqslant 2$. Moreover, from the proof of Lemma 2(b) we get that an extremal set $A$ satisfies

$$
\begin{aligned}
3 k-\frac{a+b+c}{2} & =R_{3}(A)=|\operatorname{Diff}(A)| \leqslant \frac{1}{2} \sum_{0 \leqslant i \neq j \leqslant 2}\left(\left|D_{i}(A) \cup D_{j}(A)\right|\right) \\
& \leqslant \frac{1}{2}((2 k-a)+(2 k-b)+(2 k-c))=3 k-\frac{a+b+c}{2} .
\end{aligned}
$$

We conclude that for an extremal set $A$ equalities (i), (ii) and (iii) are true. In order to check part (iv), we may assume without loss of generality that $i=0, j=1$ and we will show that if $\left|D_{0}(A) \cup D_{1}(A)\right|=2 k-a$, then for every line $\ell$ parallel to the segment $\left[e_{0}, e_{1}\right]$ the set $A \cap \ell$ is an arithmetic progression of difference $e_{1}-e_{0}$.

Let us recall that $A_{h}=A \cap(y=h)$ and $A_{ \pm h}=A_{h} \cup A_{-h}$. From the proof of Lemma 2 we get that (33) implies: 
and

(i) $\left|D_{0}\left(A_{0}\right) \cup D_{1}\left(A_{0}\right)\right|=2\left|A_{0}\right|-1$, if $A_{0} \neq \emptyset$

(ii) $\left|D_{0}\left(A_{ \pm h}\right) \cup D_{1}\left(A_{ \pm h}\right)\right|=2\left|A_{ \pm h}\right|-2$, for every integer $h>0$ such that $A_{ \pm h} \neq \emptyset$. In view of Proposition 2 and assertion (c) of Lemma 1 we get that for every line $\ell$ parallel to the segment $\left[e_{0}, e_{1}\right]$ the set $A \cap \ell$ is an arithmetic progression of difference $e_{1}-e_{0}$.

For the next two results we shall use the notations of Lemma 3 . Let $K$ be a finite nonempty subset of $\mathbb{Q}^{2}$. Assume that $K$ lies on $a \geqslant 1$ lines parallel to $y=0$, on $b \geqslant 1$ lines parallel to $x=0$ and on $c \geqslant 1$ lines parallel to $x+y=0$. If $P=(x, y)=K \backslash K^{\prime}$, we say that $P$ is a boundary point of $K$ and write $P \in B d(K)$ (see Figure 2).

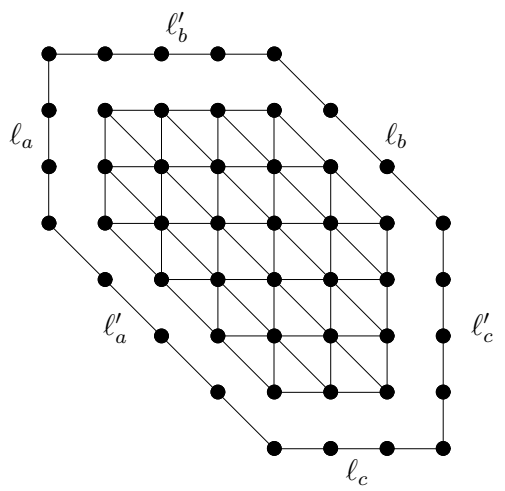

Figure 2. The set $H(3)$ and the boundary $B d(H(4))=H(4) \backslash H(3)$.

Assertion 3. If $k=|K|=\frac{(a+b+c)^{2}}{12}=3 \alpha^{2}$, then

(i) $a \geqslant 2, b \geqslant 2, c \geqslant 2$ and $\max \{a, b, c\}<\frac{a+b+c}{2}$.

(ii) the set of all boundary points of $K$ satisfies $|B d(K)|=a+b+c-3$.

(iii) the set $K^{\prime}=K \backslash B d(K)$ lies on $a^{\prime}=a-2$ lines parallel to $y=0$, on $b^{\prime}=b-2$ lines parallel to $x=0$ and on $c^{\prime}=c-2$ lines parallel to $x+y=0$. Moreover, if $K^{\prime} \neq \emptyset$, then $\max \left\{a^{\prime}, b^{\prime}, c^{\prime}\right\}<\frac{a^{\prime}+b^{\prime}+c^{\prime}}{2}$ and if $K^{\prime}=\emptyset$, then $a=b=c=2, k=3$.

(iv) $\left|K^{\prime}\right|=\frac{\left(a^{\prime}+b^{\prime}+c^{\prime}\right)^{2}}{12}=3(\alpha-1)^{2}$.

Proof. If $\min \{a, b, c\}=1$, then $a+b+c$ is odd and this contradicts $k=|K|=$ $\frac{(a+b+c)^{2}}{12}$. Therefore, in what follows we will assume that

$$
\max \{a, b, c\}=a \geqslant b \geqslant c \geqslant 2 .
$$

We prove first that the set $K$ satisfies

$$
\max \{a, b, c\}<\frac{a+b+c}{2} .
$$

To the contrary, if $a=\max \{a, b, c\} \geqslant \frac{a+b+c}{2}$, then $b c \geqslant k=\frac{(a+b+c)^{2}}{12} \geqslant \frac{(b+c)^{2}}{3}$ implies $b^{2}-b c+c^{2} \leqslant 0$, which is impossible for a nonempty set $\frac{12}{K}$. 
We clearly have

$$
a^{\prime} \leqslant a-2, b^{\prime} \leqslant b-2, c^{\prime} \leqslant c-2, a^{\prime}+b^{\prime}+c^{\prime} \leqslant a+b+c-6 .
$$

Case 1. Assume that $\max \left\{a^{\prime}, b^{\prime}, c^{\prime}\right\} \geqslant \frac{a^{\prime}+b^{\prime}+c^{\prime}}{2}$. We shall prove that

$$
a^{\prime}=b^{\prime}=c^{\prime}=0, K^{\prime}=\emptyset, a=b=c=2, k=|K|=3 \text {. }
$$

Using (36) we get $\max \left\{a^{\prime}, b^{\prime}, c^{\prime}\right\} \leqslant \max \{a, b, c\}-2=a-2$ and Lemma 4(a) implies $k^{\prime}=\left|K^{\prime}\right| \leqslant \frac{(a-2)^{2}}{4}$. From inequality (19) we get that

$$
\frac{(a+b+c)^{2}}{12}=k \leqslant k^{\prime}+(a+b+c-3) \leqslant \frac{(a-2)^{2}}{4}+(a+b+c-3)
$$

and so

$$
(a+b+c-6)^{2} \leqslant 3(a-2)^{2} .
$$

Note that $a=\max \{a, b, c\}<\frac{a+b+c}{2}$ and $a+b+c$ is even imply that $b+c \geqslant a+2$ and we obtain that $4(a-2)^{2} \leqslant(a+b+c-6)^{2} \leqslant 3(a-2)^{2}, a=\max \{a, b, c\}=2$; we conclude that $a=b=c=2, k=3, a^{\prime}=b^{\prime}=c^{\prime}=0$, which proves (37).

Case 2. Assume that $\max \left\{a^{\prime}, b^{\prime}, c^{\prime}\right\}<\frac{a^{\prime}+b^{\prime}+c^{\prime}}{2}$. Using (19) and Lemma 4(b) for the set $K^{\prime}$ we obtain

$$
\frac{(a+b+c)^{2}}{12}=k \leqslant k^{\prime}+(a+b+c-3) \leqslant \frac{\left(a^{\prime}+b^{\prime}+c^{\prime}\right)^{2}}{12}+\frac{\delta^{\prime}}{4}+(a+b+c-3) .
$$

Note that if $a^{\prime}+b^{\prime}+c^{\prime} \leqslant a+b+c-7$, then $k \leqslant \frac{(a+b+c-7)^{2}}{12}+\frac{1}{4}+(a+b+c-3)<$ $\frac{(a+b+c)^{2}}{12}$, which contradicts the hypothesis $k=\frac{(a+b+c)^{2}}{12}$. Therefore, inequalities (36) imply

$$
\begin{aligned}
& a^{\prime}+b^{\prime}+c^{\prime}=a+b+c-6, a^{\prime}=a-2, b^{\prime}=b-2, c^{\prime}=c-2, \\
& a^{\prime}+b^{\prime}+c^{\prime} \equiv a+b+c \equiv 0(\bmod 6), \delta^{\prime}=\delta=0
\end{aligned}
$$

and we conclude that

$\frac{(a+b+c)^{2}}{12}=k \leqslant k^{\prime}+(a+b+c-3) \leqslant \frac{\left(a^{\prime}+b^{\prime}+c^{\prime}\right)^{2}}{12}+(a+b+c-3)=\frac{(a+b+c)^{2}}{12}$

and so

$$
k-k^{\prime}=|B d(K)|=a+b+c-3, k^{\prime}=\frac{\left(a^{\prime}+b^{\prime}+c^{\prime}\right)^{2}}{12} .
$$

Assertion 3 is proved. 
Using the same notations we have

Assertion 4. If $k=|K|=\frac{(a+b+c)^{2}}{12}=3 \alpha^{2}$, then $a=b=c=2 \alpha$.

Proof. We shall use induction on $\min =\min \{a, b, c\} \geqslant 2$. Note that $\min \{a, b, c\}=$ 1 is impossible, because of assertion 3(i).

If $\min =2$, then we may assume that $2=c \leqslant b \leqslant a$ and we get

$$
k=|K|=\frac{(a+b+c)^{2}}{12} \leqslant 2 b .
$$

These inequalities have only one solution, namely $a=b=c=2$. Indeed, if $a \geqslant 4$, then $24 b \geqslant(a+b+c)^{2} \geqslant(b+6)^{2}$ implies $a \geqslant b=6, c=2$ and this contradicts (38). If $a=3$, then $(a, b, c)=(3,3,2)$ or $(a, b, c)=(3,2,2)$ and this contradicts $a+b+c \equiv 0(\bmod 6)$. We get that $a=b=c=2$.

Let $\min \geqslant 3$ and assume that Assertion 4 holds for smaller values of min. Using Assertion 3 (iii) and (iv) we get that

$$
k^{\prime}=\left|K^{\prime}\right|=\frac{\left(a^{\prime}+b^{\prime}+c^{\prime}\right)^{2}}{12}=3(\alpha-1)^{2} ;
$$

moreover, the set $K^{\prime}=K \backslash B d(K)$ lies on $a^{\prime}=a-2 \geqslant 1$ lines parallel to $y=0$, on $b^{\prime}=b-2 \geqslant 1$ lines parallel to $x=0$ and on $c^{\prime}=c-2 \geqslant 1$ lines parallel to $x+y=0$. Note that $\min \left\{a^{\prime}, b^{\prime}, c^{\prime}\right\}=1$ is impossible, in view of assertion 3(i). By the induction hypothesis for the set $K^{\prime}$ we obtain $a^{\prime}=b^{\prime}=c^{\prime}=2(\alpha-1)$ and thus $a=b=c=2 \alpha$. Assertion 4 is proved.

We shall use the following definition. We say that a planar $S \subseteq \mathbb{Q}^{2}$ is an $\alpha$-regular set with respect to the basis $B=\left\{e_{0}, e_{1}, e_{2}\right\}$ if:

(a) $|S|=3 \alpha^{2}$,

(b) $S$ lies on $a=2 \alpha$ lines parallel to the segment $\left[e_{0}, e_{1}\right]$, on $b=2 \alpha$ lines parallel to the segment $\left[e_{0}, e_{2}\right]$ and on $c=2 \alpha$ lines parallel to the segment $\left[e_{1}, e_{2}\right]$.

(c) For every line $\ell$ parallel to the segment $\left[e_{i}, e_{j}\right], 0 \leqslant i \neq j \leqslant 2$, the set $S \cap \ell$ is an arithmetic progression of difference $e_{i}-e_{j}$.

Note that assertions $1,2,3$ and 4 imply that every extremal set $A$ is an $\alpha$-regular set. Moreover, if $f_{0}$ is an arbitrary point on the plane and $f_{1}$ and $f_{2}$ are defined by

$$
\left(f_{i}=f_{0}+\frac{e_{i}}{2}, i=1,2\right) \text { or }\left(f_{i}=f_{0}-\frac{e_{i}}{2}, i=1,2\right),
$$

then the affine isomorphism of the plane $T$ that maps $e_{i}$ to $f_{i}, i=1,2$ defines an $\alpha$-regular set denoted by

$$
H\left(\alpha, f_{0}, f_{1}, f_{2}\right)=T(H(\alpha)) .
$$

For example, (see Figure 3) if $f_{0}=\left(\frac{1}{2}, \frac{1}{2}\right), f_{1}=\left(0, \frac{1}{2}\right), f_{2}=\left(\frac{1}{2}, 0\right)$, this set contains all the lattice points $P=(x, y) \in \mathbb{Z}^{2}$ such that

$$
-\alpha<x, y, x+y \leqslant \alpha .
$$




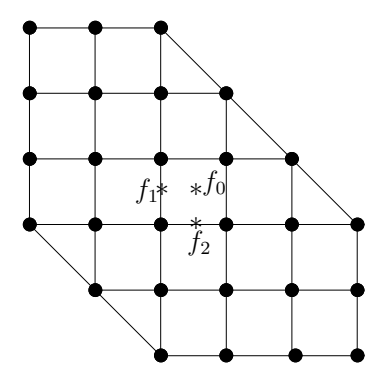

Figure 3 . The set $H\left(\alpha, f_{0}, f_{1}, f_{2}\right), \alpha=3$.

In order to conclude the proof of assertion (b) of Theorem 1 it is enough to check

Assertion 5. For every $\alpha$-regular set $S$ there are three non-collinear points $f_{0}, f_{1}, f_{2}$ such that $S=H\left(\alpha, f_{0}, f_{1}, f_{2}\right)$.

Proof. We shall use induction on $\alpha$.

For $\alpha=1$ the set $S$ contains three points and is affine isomorphic to $H(1)=$ $\{(-1,1),(1,-1),(1,1)\}$.

Let $\alpha \geqslant 2$ and assume that Assertion 5 holds for smaller values for $\alpha$. Let $S$ be an $\alpha$-regular set. Clearly $S=3 \alpha^{2}=\frac{1}{12}(a+b+c)^{2}$. We note first that

$$
S^{\prime}=S \backslash B d(S)
$$

is an $(\alpha-1)$ - regular set. Indeed, Assertion 3 implies that: $|B d(S)|=a+b+c-3=$ $6 \alpha-3,\left|S^{\prime}\right|=3(\alpha-1)^{2}$ and the set $S^{\prime}$ lies on $a^{\prime}=2 \alpha-2$ lines parallel to $y=0$, on $b^{\prime}=2 \alpha-2$ lines parallel to $x=0$ and on $c^{\prime}=2 \alpha-2$ lines parallel to $x+y=0$. Moreover, the third property of an $\alpha$-regular set is still valid for the set $S^{\prime}$. By the induction hypothesis, we get that the set $S^{\prime}$ is equal to $S^{\prime}=H\left(\alpha-1, f_{0}, f_{1}, f_{2}\right)$. The set $S$ is obtained from the set $S^{\prime}$ by adding the $6 \alpha-3$ points of $B d(S)$. This can be done in an unique way, in view that $S$ and $S^{\prime}$ satisfy both condition (c). We conclude that $S=H\left(\alpha, f_{0}, f_{1}, f_{2}\right)$.

\section{Some final remarks}

This section contains some concluding remarks and open problems.

4.1. Theorem 1 describes the structure of extremal sets $A$ such that $k=|A|=3 \alpha^{2}$. We conjecture that an extremal set $A$ with $3(\alpha-1)^{2}<k<3 \alpha^{2}$ is included in a set isomorphic to $H(\alpha)$.

4.2. It would be interesting to generalize Theorem 1 and Propositions 1 and 2 to the general case $s \geqslant 4$. More precisely, we should obtain sharp upper estimates 
for $R_{s}(A)=\left|D_{0} \cup D_{1} \cup D_{2} \cup \ldots \cup D_{s-1}\right|$ and to describe the structure of extremal sets $A^{*}$.

4.3. We conjecture that an isomorphic projection $P: \mathbb{Z}^{2} \rightarrow \mathbb{Z}$ maps the set $H(\alpha) \subseteq \mathbb{Z}^{2}$ into a set of integers $A^{*}=P(H(\alpha))$ that is the extremal set for the problem

$$
R_{3}(k)=\max \left\{R_{3}(A): A \subseteq \mathbb{Z},|A|=k\right\} .
$$

\section{References}

[1] J. Bourgain, On the dimension of Kakeya sets and related maximal inequalities, Geom. Funct. Anal. 9 (1999), no.2, 256-282.

[2] N.H. Katz and T. Tao, Bounds on arithmetic progressions and applications to the Kakeya conjecture, Mathematical Research Letters 6 (1999), 625-630.

Addresses: G.A. Freiman, School of Mathematical Sciences, Tel Aviv University, Tel Aviv 69978, Israel

Y.V. Stanchescu, The Open University of Israel, Raanana 43107, Israel and

Afeka Academic College, Tel Aviv 69107, Israel

E-mail: grisha@post.tau.ac.il; ionut@openu.ac.il and yonis@afeka.ac.il

Received: 6 December 2006 